\title{
Abstract
}

Acute kidney injury (AKI) is a common complication of acute illness. It is associated with significant morbidity and mortality as well as a high cost to healthcare systems. There are a broad range of causes of AKI which should be considered in a systematic fashion to avoid missing multiple potential causative factors. These include pre-renal causes from hypovolaemia, intrinsic renal causes such as glomerular diseases and post-renal obstructive causes. In the intensive care unit, two-thirds of AKI cases result from renal hypoperfusion, sepsis, contrast and nephrotoxic agents; up to $5 \%$ will require renal replacement therapy. Modalities of renal replacement therapy include intermittent haemodialysis, peritoneal dialysis and continuous haemofiltration. Continuous haemofiltration is usually favoured in the intensive care setting as it has greater haemodynamic stability and greater capacity to extract fluid from patients with fluid overload. Anticoagulation is recommended with haemodialysis and haemofiltration and systemic heparin, regional citrate or zero anticoagulation are the usual options.

\section{Acute Kidney Injury}

\section{Current definition and classification}

Acute kidney injury (AKI) is a clinical syndrome characterised by a sudden decline in glomerular filtration rate (GFR) sufficient to decrease elimination of nitrogenous waste products and uraemic toxins. Kidney Disease: Improving Global Outcomes (KDIGO) defines AKI as any of the following: Increase in serum creatinine by $(\geq 26.5 \mu \mathrm{mol} / \mathrm{l}$ within 48 hours; increase in serum creatinine to $\geq 1.5$ times baseline, which is known or presumed to have occurred within the prior 7 days, or urine volume $<0.5 \mathrm{ml} / \mathrm{kg} / \mathrm{h}$ for 6 hours. AKI is staged for severity according to the following criteria in table 1.

Table 1. Staging of AKI (KDIGO composite staging)

\begin{tabular}{|c|c|c|c|}
\hline \multicolumn{4}{|c|}{ Acute Kidney Injury Network (AKIN) and RIFLE classifications of Acute Kidney Injury } \\
\hline AKIN Staging & \multirow[t]{2}{*}{ Urine output } & \multicolumn{2}{|l|}{ RIFLE } \\
\hline Serum Creatinine & & Class & $\begin{array}{l}\text { Serum creatinine or } \\
\text { GFR }\end{array}$ \\
\hline $\begin{array}{l}\text { Stage } 1: \text { Increase of } \\
\times 1.5 \text { from baseline or } \\
\geq 26.5 \mu \mathrm{mol} / \mathrm{l}\end{array}$ & $<0.5 \mathrm{ml} / \mathrm{kg} / \mathrm{h}$ for $>6 \mathrm{hr}$ & Risk & $\begin{array}{l}\text { Increase in serum } \\
\text { creatinine } \times 1.5 \text { or } \\
\text { GFR decrease }>25 \%\end{array}$ \\
\hline
\end{tabular}




\begin{tabular}{|c|c|c|c|}
\hline $\begin{array}{l}\text { Stage 2: Increase of } \\
\text { x2 from baseline }\end{array}$ & $\begin{array}{l}<0.5 \mathrm{ml} / \mathrm{kg} / \mathrm{h} \text { for }>12 \\
\mathrm{hr}\end{array}$ & Injury & $\begin{array}{l}\text { Serum creatinine x2 } \\
\text { or GFR decreased } \\
>50 \%\end{array}$ \\
\hline \multirow[t]{3}{*}{$\begin{array}{l}\text { Stage 3: Increase of } \\
\times 3 \text { from baseline, or } \\
\geq 354 \mu \mathrm{mol} / / \text { with an } \\
\text { acute increase of at } \\
\text { least } 44 \mu \mathrm{mol} / / \text { or on } \\
\text { RRT }\end{array}$} & $\begin{array}{l}<0.3 \mathrm{ml} / \mathrm{kg} / \mathrm{h} \text { for } 24 \mathrm{~h} \\
\text { or anuria for } 12 \mathrm{hr}\end{array}$ & Failure & $\begin{array}{l}\text { Serum creatinine } x 3 \text {, } \\
\text { or serum creatinine } \\
\geq 354 \mu \mathrm{mol} / / \text { with an } \\
\text { acute rise }>44 \mu \mathrm{mol} / / \\
\text { or GFR decreased } \\
>75 \%\end{array}$ \\
\hline & & Loss & $\begin{array}{l}\text { Persistent acute } \\
\text { renal failure = } \\
\text { complete loss of } \\
\text { kidney function for } \\
\text { longer than } 4 \text { weeks }\end{array}$ \\
\hline & & $\begin{array}{l}\text { End-stage kidney } \\
\text { disease }\end{array}$ & ESRD $>3$ months \\
\hline
\end{tabular}

\section{Aetiology}

There are a broad range of causes of developing AKI. Differential diagnosis must be considered in a systematic fashion to avoid missing multiple factors that may be contributing to the condition. Traditionally, AKI is divided into pre-renal, renal, and post-renal causes. Pre-renal causes include hypovolaemia or a decreased effective arterial volume. Intrinsic renal causes can be considered under different anatomic components of the kidney (vascular supply; glomerular, tubular and interstitial disease). Post-renal obstructive renal failure is usually diagnosed by urinary tract dilation on renal ultrasound. In the hospital setting, pre-renal and acute tubular necrosis (ATN) account for the majority of AKI cases. These are also often superimposed on pre-existing chronic kidney disease (CKD). In the intensive care unit, two-thirds of cases of $\mathrm{AKI}$ are a result of the combination of impaired renal perfusion, sepsis and nephrotoxic agents.

\section{Prevalence and outcome}

Uncomplicated ATN typically recovers in 2-3 weeks. This could however be complicated by superimposed renal insults. Episodes of hypotension induced by haemodialysis may lead to additional ischaemic lesions and delay renal functional recovery, especially in patients who have multiple co-morbidities. It is becoming increasingly recognised that even lesser 
degrees of kidney injury have important implications for health as AKI represents a heterogenous clinical syndrome with multiple causes rather than one disease.

The incidence of $\mathrm{AKI}$ in unselected hospitalised patients has been estimated to be $18 \%$, and AKI accounts for $1-4 \%$ of all hospital admissions. AKI has the highest incidence among those aged 65 and older. Patients with eGFR $<30 \mathrm{ml} / \mathrm{min} / 1.73 \mathrm{~m}^{2}$ have a $30-40$ fold higher risk of developing $\mathrm{AKI}$. AKI is associated with high costs and adverse clinical outcomes, including increased mortality, length of stay and development or progression of CKD. The cost of AKI to the NHS in England in 2014 was estimated to be $£ 1.02$ billion. The number of cases of AKI increased from over 600,000 in 2001 to over 3 million in 2011, although mortality has significantly decreased from $21.9 \%$ to $9.1 \%$ in 2001 . The mortality of AKI ranges from $8.1 \%$ in patients with $\mathrm{AKI}$ stage 1 to $33 \%$ in $\mathrm{AKI}$ stage 3 .

The incidences of CKD and ESRD after AKI were 25.6 and 8.6 per 100 person-years respectively. The annual absolute risk for developing ESRD after an episode of AKI ranges from $0.6 \%$ to $1.2 \%$ in those with mild AKI compared to $9 \%$ in those with pre-existing CKD. Patients should be followed up at 3 months after AKI for resolution, new-onset or worsening of pre-existing CKD.

\section{AKI in the Intensive Care Unit}

AKI is an independent risk factor for death and up to $40 \%$ of ICU patients develop AKI. Up to $5 \%$ of ICU patients require renal replacement therapy. Factors that exacerbate acute kidney injury include hypovolaemia in the peri-operative period, repeated exposure to contrast and nephrotoxic agents, and inflammatory states. Mortality is attributed to infection, haemorrhage or persistent shock despite optimal care.

\section{Acute management of AKI}

Primary prevention and early diagnosis of $\mathrm{AKI}$ are essential in improving outcomes and preventing residual effects of kidney damage. All patients should have a risk assessment to identify and reverse risk factors. Major risk factors for AKI include those with older age (>75 years), diabetes, hypertension, sepsis, malignancy, surgical patients, pre-existing cardiac disease.

A key factor in preventing $\mathrm{AKI}$ is haemodynamic stabilisation with optimisation of cardiac output and blood pressure to ensure adequate renal perfusion. Adequate volume expansion is essential in decreasing risk of $\mathrm{AKI}$ in the perioperative period in the initial phase, but this needs to be balanced with preventing the undesirable side effects of fluid accumulation and overload. It can be particularly challenging to manage patients with severe congestive cardiac failure or diastolic dysfunction, where renal perfusion is inadequate despite normal 
volume status. Excessive fluid replacement is tolerated poorly in this patient group and may precipitate pulmonary oedema.

The International Guidelines for Sepsis Management by the Surviving Sepsis campaign recommends initial fluid resuscitation with crystalloids for a minimum of $30 \mathrm{ml} / \mathrm{kg}$ and adding albumin in patients who continue to require substantial amounts of crystalloid to maintain adequate mean arterial pressure (MAP). Fluid challenge should be continued for as long as haemodynamic parameters continue to improve. Late and aggressive fluid resuscitation in critically ill patients have been associated with worse renal outcomes and increased mortality. The Fluid and Catheter Treatment Trial (FACTT) indicates that after initial resuscitation, a conservative approach to fluid administration is associated with faster weaning from mechanical ventilation and decreased ICU length of stay without any deterioration of kidney function or worse outcomes in patients with acute lung injury. The Vasopressin and Septic Shock Trial (VASST) study showed that the best survival outcomes are with a positive fluid balance of approximately 3 litres within 12 hours. Therefore, a liberal fluid approach appears to be beneficial in the first hours of shock, whilst a conservative approach following resolution of shock is preferred.

KDIGO AKI guidelines recommend isotonic crystalloids instead of colloids for intravascular expansion in patients at risk of AKI. In critically ill patients receiving mechanical ventilation, respiratory changes in left ventricular stroke volume can predict fluid responsiveness. In hypovolaemic patients, positive-pressure ventilation may induce a fall in venous return and lead to an increase in cardiac output.

\section{Prevention of Drug and Nephrotoxin-Induced Acute Kidney Injury}

Risk factors for developing nephrotoxicity include age older than 60 years, pre-existing CKD, volume depletion, diabetes, heart failure, and sepsis. Drug monitoring of nephrotoxins is crucial in at-risk patients. Preventative measures include correctly estimating the GFR before initiation of therapy, adjusting the dosage of potential nephrotoxins, and monitoring renal function during therapy.

\section{Contrast-induced nephropathy (CIN)}

AKI secondary to contrast nephropathy (defined as increase in creatinine of $>44 \mu \mathrm{mol} / \mathrm{l}$ ) typically occurs in patients with underlying renal impairment and is rarely seen in patients with normal renal function. It may occur with intravenous and intra-arterial contrast, but not with oral contrast. The incidence of CIN is $20 \%$ in patients with creatinine levels of more than $176 \mu \mathrm{mol} / \mathrm{l}$ and $50 \%$ when levels are more than $440 \mu \mathrm{mol} / \mathrm{l}$. Other risk factors include diabetic nephropathy, advanced age (older than 75 years), congestive heart failure, volume 
depletion, and high or repetitive doses of radiocontrast agents. The KDIGO AKI guidelines recommend volume expansion with either isotonic sodium chloride or sodium bicarbonate solutions in patients at risk of $\mathrm{Cl}-\mathrm{AKI}$ unless there are contraindications. Measures to reduce AKI risk should be implemented in those with eGFR $<45 \mathrm{ml} / \mathrm{min} / 1.73 \mathrm{~m}^{2}$.

\section{Treatment of AKI}

In recent years, there has been a lot of focus on improving timely diagnosis and earlier recognition of $\mathrm{AKI}$ as it is becoming increasingly recognised that serum creatinine is a relatively late indicator of renal injury. Initial management of $A K I$ includes thorough assessment of the cause of renal dysfunction and the volaemic status. Nephrotoxic agents should be avoided and exposure to intravascular radiocontrast dye needs to be carefully rationalised. Gadolinum-based contrast agents should be avoided due to the risk of developing nephrogenic systemic fibrosis. Antimicrobial agents such as aminoglycosides, amphotericin and acyclovir should be avoided when possible, with doses calculated according to renal function.

Care needs to be taken to recognise and manage treatment of $\mathrm{AKI}$ complications. When fluid overload occurs in patients with AKI, fluid intake should be restricted with a trial of diuretics before dialysis initiation. Morphine and nitrates can be used to alleviate respiratory symptoms. When fluid overload cannot be corrected in a timely manner with medical management, positive-pressure ventilation may need to initiated with or without endotracheal intubation and dialysis.

Hyperkalaemia is a frequent complication of $\mathrm{AKI}$ and can be managed by administering parenteral glucose and insulin infusions and sodium bicarbonate to promote shift of potassium into the intracellular space. Dialysis interventions for the treatment of AKI should be considered in the broader clinical context with trends of laboratory tests rather than one single reading and with due consideration of whether or not the condition can be managed by initiation of RRT.

Nutritional support is an important but frequently neglected aspect in the management of AKI. Patients with AKI have an increased risk of protein-energy malnutrition because of poor nutritional intake and high catabolic rate. Patients with AKI should receive a basic intake of 0.8-1.0g of protein $/ \mathrm{kg} /$ day and a total energy intake of $20-30 \mathrm{kcal} / \mathrm{kg} / \mathrm{day}$. In patients on RRT, 1.0 to $1.5 \mathrm{~g}$ of protein per $\mathrm{kg} /$ day should be given up to a maximum of $1.7 \mathrm{~g} / \mathrm{kg} / \mathrm{day}$ in hypercatabolic patients. 


\section{Diagnostic approach to AKI}

Prior creatinine concentration and evidence of underlying kidney should be evaluated from the medical history and hospital records. Particular attention should be given to the medication history especially any over the counter medications. Painless haematuria suggest glomerulonephritis or a genitourinary malignancy, while painful haematuria is more consistent with obstruction. Physical examination, especially assessment of volume status is particular helpful. Trends in daily intake and output volumes assist in determining the extracellular fluid volume of the critically ill patient. Abdominal examination can reveal important information, such as a distended bladder suggesting lower urinary tract obstruction, or a tense distended abdomen which may point towards abdominal compartment syndrome. Intra-abdominal pressure can be measured in the ICU and a diagnosis of abdominal compartment syndrome made when pressures exceed $20 \mathrm{mmHg}$.

Fever, skin rash and arthralgias may point towards sings of a systemic disease such as systemic lupus erythematous, vasculitis, endocarditis, or a drug allergy with acute interstitial nephritis. A leukocytoclastic rash on the lower extremities could be Henoch-Schonlein purpura or cryoglobulinamia in a patient with Hepatitis C. In patients with recent aortic catheterisation, it is important to look for livedo reticularis or a discoloured toe as they could help with diagnosing cholesterol or artheromatous emboli.

\section{Diagnostic Workup}

Evaluation of urine volume, urinary sediment, and serum and urine basic metabolic panel is important for the diagnosis of AKI.

Urinalysis is one of the key tests to evaluate kidney disease although this is less helpful in the ICU setting as most urine specimens are collected from a urinary catheter. Ultrasound is commonly used to rule out obstructive renal disease. Contrast CTs are often needed to identify retroperitoneal fibrosis, renal vein thrombosis, renal infarction or nephrocalcinosis as cause of acute kidney injury. It is important to remember the risks of contrast-induced nephropathy in patients with renal impairment as well as the risk of nephrogenic systemic fibrosis in patients exposed to gadolinium.

A renal biopsy should be considered for patients in whom pre-renal and post-renal AKI have been excluded and if a cause of intrinsic renal disease is suspected, yet remains unclear. Examples where it would be useful include rapidly proliferative glomerulonephritis (RPGN), vasculitis, SLE, and acute interstitial nephritis. As kidney biopsies pose a significant risk it is crucial to consider if the pathological diagnosis would ultimately change management. 


\section{AKI biomarkers and early detection of AKI}

Increasingly research efforts have been focussed on the early detection of AKI as it has been known to improve outcomes. Novel serum and urine biomarkers, such as kidney injury molecule 1 (KIM-1), neutrophil gelatinase-associated lipocalin (NGAL), cystatin C, interleukin (IL-18), urinary cystatin $C$ and others suggest potential at prognostification and identifying specific causes of AKI.

\section{Chronic kidney disease}

Chronic kidney disease (CKD) is defined as abnormalities of kidney structure or function, present for $>3$ months, with implications for health. The criteria for diagnosis of CKD include albuminuria, urine sediment abnormalities, electrolyte and other abnormalities due to tubular disorders, abnormalities detected by histology, structural abnormalities detected by imaging, history of kidney transplantation, or an estimated eGFR below $60 \mathrm{ml} / \mathrm{min} / 1.73 \mathrm{~m}^{2}$ persisting for 3 months or more irrespective of the cause. CKD should be classified based on GFR category ( $\mathrm{G} 1$ to $\mathrm{G} 5$, with $\mathrm{G} 3$ split into $3 \mathrm{a}$ and $3 \mathrm{~b}$ ) and albuminuria category (A1, A2 and A3 based on urinary albumin/creatinine ratio in an early morning spot urine sample).

Clarifications should be made to include the suffix $T$ for patients with renal allografts and $D$ to identify CKD stage 5 patients on dialysis.

\section{Renal replacement therapy}

In the face of untreated acute renal failure, the patient will die from accumulation of unexcreted solutes (urea, potassium, hydrogen ions) or fluid overload. It is only in the last century that we have been able to fully understand and treat this. The 20th century has seen the rapid rise and subsequent refinement of several modalities of renal replacement therapy. In the 1940s Dr Willem Kolff developed the first drum dialysis machines for haemodialysis. In the same decade Dr Jacob Fine developed the process of peritoneal dialysis. In the 1960s Dr Berland Scribner developed continuous arteriovenous haemofiltration and Dr Bernard Canaud developed continuous venovenous haemofiltration in the 1980s. Since these discoveries, the processes have been further refined to improve safety, efficiency and reliability. As a result of these advances, we now have a range of options for the provision of renal replacement in acute renal failure.

\section{Intermittent Haemodialysis}

Once the prime modality of renal replacement in the acute and chronic setting, this has become far less popular in critical care in favour of continuous modes of renal replacement. 
It remains far more popular outside of critical illness due to the capacity to be run intermittently rather than continuously thereby liberating the patient from the machine for long periods of time.

The principle of haemodialysis is the passing of blood over a semipermeable membrane. A dialysate is then passed on the other side of the semipermeable membrane. The dialysate is passed in the opposite direction to the blood flow (countercurrent flow) to maximise the concentration gradient across the membrane and enhance the efficiency of the system. A dialysate is a solution of specially treated mains-supply water with an added concentrate of sodium, calcium, potassium, magnesium, chloride, acetate, citrate, bicarbonate and glucose. Mains supply water is required due to high volumes of liquid involved in the process (500$800 \mathrm{ml} / \mathrm{min}$ of dialysate flow countercurent against a blood flow of $250-400 \mathrm{ml} / \mathrm{min}$ ). Water and low molecular weight substances (roughly 10kDa or less but dependent on the device) diffuse across the membrane. This process requires the presence of an osmotic gradient between the two sides of the membrane (blood and dialysate). Brownian (random) particle movement will favour a bulk movement of solute (small particles such as urea) to the side of high concentration to low concentration and water alongside it. Therefore, water will pass across the membrane only if the dialysate is suitably hypertonic compared to blood and electrolytes (such as sodium and potassium) will only be removed from the blood if the dialysate contains lower concentrations of the electrolytes than the blood. This process of solute removal if referred to as diffusion.

Solute clearance is greater than normal renal clearance and it is possible for a session of dialysis lasting 2-4 hours to provide adequate renal replacement for 48 hours or more. This ensures that the treatment is intermittent and the patient can be disconnected form the circuit for prolonged periods of time. It is possible to run slower flows of dialysate through the device $(100 \mathrm{ml} / \mathrm{min}$ flow), but this reduces the efficiency of the solute clearance and necessitates more prolonged sessions; this low efficiency setup is called SLED (sustained low efficiency dialysis).

Figure 1 gives a schematic of the process of dialysis. The figure is simplified, instead of a single tube constructed of a semipermeable membrane, modern dialysers consist of hundreds of small hollow fibres encased in a larger cylinder. This has the benefit of vastly increasing the surface area of membrane (usually $0.8-2.5 \mathrm{~m} 2$ ) that blood is in contact with, thereby increasing the rate of diffusion and therefore solute clearance. Historically the membrane was composed of cellulose, although this has largely been replaced with synthetic materials such as polysulfone and polyacrylonitrile. 


\section{Peritoneal dialysis}

The peritoneum is a vascular structure covered with thin mesothelium. The structure covers the gut and other intraabdominal organs and the surface of the abdominal cavity. It has a surface area of $1-2 m 2$ which is enhanced by the microvillae on the surface of the mesothelium. Due to these properties, it is an ideal membrane for the transfer of water and low molecular weight molecules.

Dr Henry Tenchkoff developed the so-called Tenchkoff catheter in the 1960s. It remains largely unchanged today; a synthetic tube with either a flange or Dacron cuff (usually 2 Dacron cuffs) to ensure fixed placement is inserted through the anterior abdominal wall into the peritoneal cavity. This can be inserted with a Seldinger (blind) technique, laparoscopically or via open surgical approach.

A dialysate is instilled into the peritoneal cavity. It is usually comprised of sodium, calcium, magnesium, chloride, lactate, bicarbonate and glucose. Usual volume is $1.5-3 \mathrm{~L}$. The fluid is usually instilled, permitted to dwell in the peritoneum whilst solute exchange occurs and then it is expelled. This usually occurs via automated device whilst the patient is asleep overnight. A dose of dialysate can be left to dwell in the patient during the day for ongoing continuous ambulatory dialysis or the patient can be left 'dry' during the day with no indwelling fluid.

The peritoneum is a semipermeable membrane; as such, it acts akin to the membrane in a dialysis machine and allows small molecules such as urea to diffuse from the blood to the fluid across along the concentration gradient. The osmolality of the dialysate (largely due to high glucose content) also creates an osmotic pressure that drags water and solutes into the fluid via ultrafiltration.

Several factors make this method of dialysis less favourable in critically ill patients including the reduced control of fluid balance, variable solute clearance, peritoneal infection risk, protein loss and increase in abdominal pressures. Peritoneal dialysis has been demonstrated to confer a higher mortality risk as compared to continuous haemofiltration in critical care.

\section{Continuous haemofiltration}

This method of renal replacement therapy is the most recent to be developed as compared to other modalities, but it has risen into position as the most preferred method of renal replacement therapy in critical care, especially in high resource countries. There are several reasons for this including: 
- improved haemodynamic stability

- greater control of $\mathrm{pH}$ and electrolyte imbalances

- greater capacity to extract fluid from the vascular compartment in fluid overloaded patients

- less rise in intracranial pressure as compared to other methods

- low infection risk

- no requirement for specific treated water supply

- simple to use and train staff to operate

Originally, the continuous haemofiltration was designed to be connected to the patient via a designated arterial cannula and venous cannula. Blood flowed through the circuit from the arterial access to venous return under mean arterial pressure. This has subsequently been replaced by dual lumen venous catheters that provide access and return ports in the same cannula and the addition of pumps within the circuit to ensure that the flow is independent of patient blood pressure and avoids the requirement for arterial vascular access. This is referred to as continuous venovenous haemofiltration (CVVHF).

The process has similarities to dialysis, insofar as blood is pumped out of the patient and passed over a semipermeable membrane where solutes and water pass through pores in the membrane. The filter (like a dialyser) is also arranged as hundreds of hollow fibres of the membrane material encased in a larger cylinder. The difference lies in the mechanism by which the solutes and water pass across the membrane. Unlike dialysis, a hydrostatic pressure is maintained within the fibres of the filter. This pressure across the membrane (so called transmembrane pressure - TMP) drives solutes and water across the membrane without the requirement of an osmotic gradient to promote it. The mechanism of driving the solutes across the membrane using pressure is referred to as convection.

The rate of ultrafiltrate production (Qf) depends upon the membrane permeability coefficient $(\mathrm{Km})$ and the TMP:

$\mathrm{Qf}=\mathrm{Km} \times \mathrm{TMP}$

Figure 2 gives a schematic of the equipment arrangement for CVVHF. Blood flow is usually static at approximately $200 \mathrm{ml} / \mathrm{min}$. A pump controlling the ultrafiltrate removal manipulates the TMP by controlling the pressure on that side of the circuit. This ensures that the ultrafiltrate flow can be maintained at a constant rate even as the membrane permeability alters (it tends to reduce over time). Another key difference between haemodialysis and haemofiltration is the removal of water and electrolytes from the patient. This is targeted by the composition of the dialysate in dialysis; in haemofiltration, water and all substances 
smaller than the pore size of the semipermeable membrane are filtered. This results in large volumes of fluid and electrolyte loss which much be replaced in the circuit. Replacement fluid can be added before the filter (predilution), after (postdilution), or a mix of both. Replacement fluid contains water and most electrolytes present in plasma. It is usually buffered with lactate and bicarbonate. Phosphate is often absent from replacement fluid and it is not uncommon for patients to require phosphate replacement when receiving haemofiltration. Predilution reduces the haematocrit of the blood and potentially increases the useable lifetime of the filter before it clots, but reduces the efficiency of the filter. Ultrafiltrate dose is prescribed according to weight $(\mathrm{ml} / \mathrm{kg} / \mathrm{hr})$. There has been virtually no demonstrated benefit from high volume haemofiltration $(35-40 \mathrm{ml} / \mathrm{kg} / \mathrm{hr}$ ) as compared to low volume haemofiltration $(20-25 \mathrm{ml} / \mathrm{kg} / \mathrm{hr})$ in acute kidney injury and low volume prescriptions are usually favoured.

It is possible to modify a CVVHF circuit to pass a dialysate over the semipermeable membrane in a countercurrent flow (see Figure 3). This is referred to as continuous venovenous haemodiafiltration (CVVHDF) and it enhances solute clearance by permitting convection and diffusion to occur.

\section{Anticoagulation}

When blood is passed in an extracorporeal circuit and exposed to synthetic materials it triggers activation of the coagulation pathways. This results in formation of blood clots within the circuit, specifically clotting and failure of the dialyser/filter. Improvements in materials have reduced this effect over time and it is possible to run both dialysis and haemofiltration circuits without anticoagulation, but it remains recommended that anticoagulation is used to reduce the risk of circuit failure.

For continuous renal replacement therapies there are several options

- Systemic anticoagulation with unfractionated heparin

- Regional anticoagulation with unfractionated heparin

- Regional anticoagulation with citrate

Systemic anticoagulation involves anticoagulating the patient and the extracorporeal circuit by adding heparin via a bolus and continuous infusion. The activated partial thromboplastin time (APTT) is checked and a target APTT ratio of 1.5-2.5 is usual. In patients with a high bleeding risk, it is possible to add the heparin in the afferent limb of the circuit, the effect can then be reversed in the efferent limb of the circuit with a calculated dose of protamine. This is referred to as regional anticoagulation, with anticoagulant effect only present in the blood 
between the heparin and protamine infusions, the blood coagulation status is normalised prior to re-entering the patient. This is now uncommonly used as patients with a bleeding risk are now usually filtered without anticoagulation or with regional citrate anticoagulation is available.

Regional citrate anticoagulation involves adding sodium citrate to the afferent limb of the circuit. This chelates calcium in the blood and inhibits coagulation in the circuit. An infusion of calcium chloride or gluconate is then added to the efferent limb of the circuit to overcome the effect of the citrate and normalise coagulation before it re-enters the patient. Serum ionised calcium levels are measures from the patient and the calcium replacement is titrated accordingly. The citrate is mostly removed via the filter, any remaining calcium/citrate complexes are then metabolised in the liver. The excess citrate amounts to a significant alkali load and specialised replacement fluids with reduced bicarbonate are needed.

\section{Key learning points:}

- Acute kidney injury has significant morbidity and mortality

- It can be cause be pre-renal, renal or post-renal causes. In the ICU setting it is often multi-factorial

- Optimising fluid management and preventing nephrotoxins is key in prevention and management of AKI

- Dialysis works via diffusion

- Filtration works via convection

- Peritoneal dialysis is inferior to haemodialysis and haemofiltration in acute renal failure but may be appropriate in a low resource setting

- Anticoagulation is recommended and systemic heparin, regional citrate or zero anticoagulation are the usual options

\section{Suggested Reading}

Kidney Disease: Improving Global Outcomes (KDIGO) Acute Kidney Injury Work Group KDIGO Clinical Practice Guideline for Acute Kidney Injury. Kidney Int Suppl. 2012;2:1-138.

Johnson RJ, Feehally J, Floege J. Comprehensive Clinical Nephrology (5th Edition) Elsevier Saunders. Philadelphia. 2015.

Daugirdas JT, Blake PG, Ing TS. Handbook of Dialysis (5 $5^{\text {th }}$ Edition). Wolters Kluwer Health. Philadelphia. 2015. 
